\title{
La photographie au révélateur littéraire : de Denis Roche à Anne-Marie Garat
}

Dominique Kunz Westerhoff

\section{(2) OpenEdition \\ 1 Journals}

Édition électronique

URL : http://journals.openedition.org/edl/582

DOI : $10.4000 /$ edl.582

ISSN : 2296-5084

Éditeur

Université de Lausanne

\section{Édition imprimée}

Date de publication : 15 décembre 2013

Pagination : 183-204

ISBN : 978-2-940331-33-8

ISSN : 0014-2026

Référence électronique

Dominique Kunz Westerhoff, "La photographie au révélateur littéraire : de Denis Roche à Anne-Marie Garat », Études de lettres [En ligne], 3-4 | 2013, mis en ligne le 15 décembre 2016, consulté le 18

décembre 2020. URL : http://journals.openedition.org/edl/582 ; DOI : https://doi.org/10.4000/edl.582

(c) Études de lettres 


\section{LA PHOTOGRAPHIE AU RÉVÉLATEUR LITTÉRAIRE: DE DENIS ROCHE À ANNE-MARIE GARAT}

Au début des années 1980, l'écrivain et photographe Denis Roche fonde la "photoautobiographie», qui dramatise la prise de vue dans un dispositif intermédial. Quelques années plus tard, Gilles Mora théorise la «photobiographie» comme un nouveau genre, proche de l'autofiction. Il en accentue la dimension narrative et fédère à cette enseigne de nombreuses démarches d'artistes contemporains. Anne-Marie Garat, que Denis Roche a publiée et qui a elle-même commenté l'œuvre rochienne, sera lue dans ce contexte et dans son inflexion romanesque apportée à la photobiographie. Entre l'essai et le roman photographique, la matérialité des images devient chez elle matière à intrigues où se tressent l'enquête technique, l'investigation historique et la quête de soi. Par là, ce sont les défauts du visible, autant que les infirmités de l'Histoire et des histoires individuelles des personnages, qui se répondent.

\section{La photo-autobiographie de Denis Roche: une dramatisation de la prise de vue}

En 1981, l'écrivain et photographe Denis Roche fait paraitre un ouvrage intitulé Légendes de Denis Roche. Essai de photo-autobiographie. Le titre constitue le leitmotiv d'une collection qui décline les «légendes" photographiques de multiples auteurs. Mais Roche déplace d'emblée la dimension narrative de l'autobiographie illustrée vers une dramatisation de l'acte photographique lui-même. La prière d'insérer fonde un nouveau genre, comme le revendique le néologisme du sous-titre, et le décrit moins comme l'histoire d'une vie que comme l'histoire d'une image: 
Que veut dire l'idée qu'un homme raconte sa vie en photos, sinon qu'il s'est fait, une fois pour toutes, à cette autre idée: qu'il n'a plus désormais affaire qu'à l'incessant et admirable aller et retour que la lumière et la forme vont entreprendre, à sa place, entre la mort et la vie, c'est-à-dire entre l'oubli et Narcisse, entre le corps qu'on immerge et la Révélation? ${ }^{1}$

Tandis que le parcours d'un homme va de la naissance à la mort, son image photographique, elle, va d'une mort symbolique («l'oubli» dans l'image latente du négatif) à une renaissance dans la "révélation" de son reflet («Narcisse»). Entre l'irradiation de la prise de vue ("la lumière»), le développement ("le corps qu'on immerge» dans le bain chimique) et le tirage (la fixation de "la forme» sur le papier), la séquence en trois temps de la représentation photographique répéterait en l'inversant le trajet biographique, jusqu'à une forme de rédemption esthétique.

Cette autonomie technique du medium suppose que l'image photographique vit en quelque sorte sa propre vie: elle fait réapparaître le "moi » en différé, se présente "à sa place» et autre, le plus souvent, que ce que l'opérateur prévoyait. Ainsi, Denis Roche multiplie les autoportraits au retardateur et se montre souvent de dos, le corps coupé hors champ, ou pris de vitesse par l'appareil:

J'entendis le déclic alors qu'à fond de course j'opérai le pivotement qui allait me permettre de me retrouver assis sereinement aux côtés de ma compagne. Comme une flèche entre les deux épaules, pas raté du tout ${ }^{2}$.

Dans ses dispositifs expérimentaux de photolittérature, Roche met en intrigue la prise de vue et fait de celle-ci, de ses hasards, l'allégorie du temps existentiel où la mort ne saurait «rater» le «moi»: «Ce qu’on photographie, c'est précisément l'instant où on fait la photo " ${ }^{3}$. Cet instant dilaté par le commentaire rétrospectif dissocie toujours l'opérateur de son "devenir image». Dans un très bel autoportrait du couple au retardateur, daté de 1979 dans les Thermes de Stabies, l'opérateur s'avance vers la femme qui le regarde, c'est-à-dire vers son destin de personnage

I. D. Roche, Légendes de Denis Roche, $4^{\mathrm{e}}$ de couverture. Sur cet ouvrage, voir M. Calle-Gruber, «Ecrit avec l'image» et J. Baetens, "Légendes de Denis Roche».

2. D. Roche, "Brève rencontre. L'autoportrait en photographie», in La disparition des lucioles, p. 100.

3. D. Roche, «Photographier», in La disparition des lucioles, p. 73. 


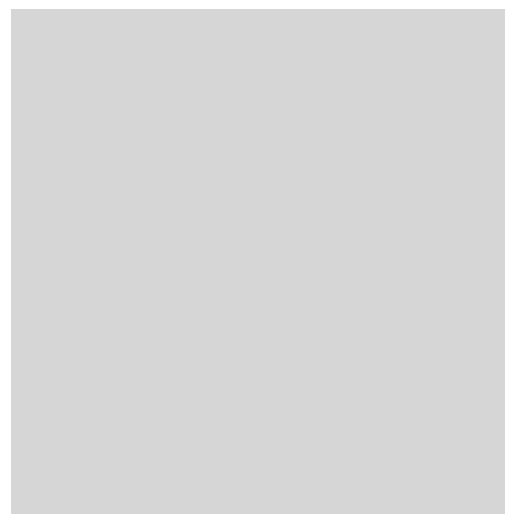

Fig. 1 - Denis Roche, «21 juillet 1979. Pompei. Thermes de Stabies ", La disparition des lucioles (réflexions sur l'acte photographique), Paris, Editions de l'Etoile, 1982.

devant prendre place aux côtés de sa compagne, elle-même immobilisée dans la pose de l'attente et déjà presque statue, à l'instar de la femme de pierre qui lui fait face et qui semble exhiber, telle un gisant dressé, la monumentalisation du couple dans le linceul de la "chambre blanche» photographique. Le chassé-croisé des positions (de dos, de face, de profil) articule la séquence d'une pétrification commune, jusqu'à la colonne de marbre qui redouble le cadre. Le mouvement de la marche lente, le décadrage du corps tronqué par le retardateur, et surtout l'angle de vue situé très bas, au niveau du sol, qui met au premier plan la terre du site ruiné, soulignent la dimension thanatographique de la photographie: l'opérateur Narcisse se découvre Orphée ${ }^{4}$ lorsqu'il entre dans l'image, et qu'il se regarde après coup dans la distance du texte ("que veut dire cette icône laconique?»). Loin d'annuler le temps dans l'instantané, le photo-autobiographe désigne une divergence immédiate entre sa propre mortalité et l'éternité à venir de son personnage dans le mémorial du couple.

Ce détournement intermédial du récit autobiographique se manifeste aussi dans le style des textes. Les «légendes» de l'essai fondateur sont plus notationnelles que narratives (date, lieu, circonstances de la prise de vue). Brèves, elles doublent les photos de famille ou les autoportraits d'une "calligraphie enchantée " 5 qui insère l'image dans des récits stratifiés au gré des investissements imaginaires de l'auteur. La légende peut d'ailleurs déterminer la prise de vue. Une photo montre ainsi Roche posant avec sa compagne Françoise Peyrot sous l'enseigne d'une coopérative ardéchoise, "Peyroche», qui combine leurs deux noms. La légende scelle cette fusion, pour inscrire l'histoire du couple dans une histoire de la langue et du site: «En langue d'oc, Peyrot veut dire roche». Prédestinés à s'unir, les

4. A ce propos, voir le bel article d'A. Buisine, "Tel Orphée...".

5. D. Roche, Légendes de Denis Roche, $4^{\mathrm{e}}$ de couverture. 
amants ne feraient, en se photographiant, que rejoindre leur personnage, occuper leur place dans une histoire d'amour déjà gravée dans le marbre, déjà écrite dans une mémoire onomastique et un génie du lieu.

Roche, en "légendeur de rêve", transforme donc le document photographique en pierre de touche d'une fiction personnelle. Parallèlement à cette mythologie surdéterminée du couple que raconte toujours l'écriture, c'est "l'histoire de la photo" qu'incline à narrer l'écrivain lorsqu'il commente les images des autres. Dans Ecrits momentanés, il s'adonne au plaisir de reconstituer la genèse de l'image, aidé ou non par la connaissance qu'il a de l'artiste, mais toujours avec une distance critique qui souligne la pulsion narrative inhérente à tout discours sur la photographie:

Ce jour-là (ainsi devrait toujours commencer «l'histoire» d'une photo, comme on dit «Il était une fois» au début d'un conte), Michel [Lamoureux] avait pris le train pour la bourgade de Pompéi, non loin de Naples ${ }^{6}$.

Dans le cas des photos d'Histoire, la rêverie rochienne hante la place invisible de l'opérateur et fait coïncider l'histoire de la photo avec le récit d'une imminence fatidique:

A chaque nouveau regard, même distrait, que je porte [à la photo d'O'Sullivan], je me laisse aller à de nouvelles rythmiques et à d'infinis dégradés de l'imaginaire, essayant de remonter le temps jusqu'à cette extrême limite où je serais enfin devenu moi-même cet homme excité qui installe, tout essoufflé, son lourd trépied surmonté de sa "caisse objective» devant cet étonnant épisode de la guerre de Sécession ${ }^{7}$.

Ces récits imaginaires, qui identifient l'instant décisif de l'image avec l'heure historique, peuvent se décliner au futur antérieur, comme dans ce commentaire d'une photo du tsar Nicolas II «nageant nu comme un ver dans les eaux glacées de la Baltique». Le corps spectral, flouté par l'eau, devient la figure anticipée d'une fin tragique:

6. D. Roche, "Sur une image de Michel Lamoureux", in Ecrits momentanés, sans pagination.

7. D. Roche, Le Boîtier de mélancolie, p. 50. 
Pour le reste, le corps paraît à demi noyé, on le dirait remonté à la surface par la mort, bras mollement écartés, torse livide comme élargi par la suspension: fantôme tout près de s'évanouir dans la tourmente de la révolution, dépouillé à jamais de lui-même ${ }^{8}$.

Porteuse de narrèmes pour l'écriture qui s'abandonne à la pente du récit («je me laisse aller...»), l'image permet au photo-autobiographe d'exacerber les techniques mêmes de la narration: l'illusion rétrospective d'un futur préfiguré, la dilatation d'une imminence, l'effet de destin des personnages transis par leur avenir inéluctable, mais aussi le hasard du déclic qui commande la mise à feu de l'obturateur, la rencontre toujours recommencée des amants marchant l'un vers l'autre, etc., tous ces topoi de la mise en intrigue sont surexposés. L'écriture «remonte le temps», et le redescend en des allers-retours narratifs autour d'un point de bascule historique que révélerait l'arrêt sur image. Métaphore de l'Histoire, la photo ferait «voir» le temps, et déroulerait son flux, à l'endroit comme à l'envers.

Le plus souvent, dans la photolittérature de Denis Roche, c'est la prise de vue elle-même qui devient le support d'un récit, parce que le rituel de sa mise en scène accentue, et brouille à la fois, ce hiatus central où le successif se renverse instantanément en spatialité dans la fixation de l'image. Les allées et venues de l'autoportraitiste au retardateur, racontées par le texte et rendues visibles dans les photos de dos, spatialisent ainsi les étapes successives de la prise de vue, tout comme elles introduisent des effets temporels (le retard du corps hors cadre, le bougé d'un mouvement) dans l'espace de l'image. "L'autoportrait à deux, c'est plus laborieux, donc il y a une mise en scène qui joue, on fait presque un montage avant la prise de vue elle-même. On se rapproche d'une idée de syntaxe " ${ }^{9}$. Cette structure séquentielle d'avant l'image, narrée par le texte et indexée par les photos, peut se construire en une véritable «syntaxe" visuelle dans ce que Roche appelle les "photolalies", à savoir des paires de photographies montrant deux états d'une même pose d'un même personnage au même endroit, parfois à une décennie d'intervalle (les doublets pouvant se sérialiser sur toute une vie). Ici, le dispositif se construit véritablement comme un récit, au sens où deux états successifs

8. Ibid., p. 96.

9. D. Roche, "Photographier», in La disparition des lucioles, p. 80. 
Fig. 2 - Denis Roche, « 26 mars 1981. Denderah, Egypte." La disparition des lucioles (réflexions sur l'acte photographique), Paris, Editions de l'Etoile, 1982. s'articulent autour d'un événement central qui est la cause de leur transformation, un événement invisible mais présent dans l'interstice des deux images: le passage du temps. C'est une fois encore le texte qui se charge de dire l'invisible: «J'ai choisi d'exposer d'abord l'écart du Temps, à la fois dans le chaos et l'ordre qui lui sont propres et au même titre qu'un écart de langage» ${ }^{10}$.

La photo-autobiographie de Denis Roche exhibe ainsi les déhiscences du temps et de l'espace qu'implique son dispositif hétérogène, d'un moment fluctuant du réel à une aire d'irradiation lumineuse.

Le langage «rouvre» le cadre de l'image, dans un écartement narratif de ses bords visuels, et la rend à une profondeur de temps. Les diagonales que trace parfois l'écriture rochienne, pour articuler des notations à une photographie ou à une série de photos, jusqu'à toucher leur pourtour, constituent le geste graphique de ce contact entre successivité narrative et image tabulaire, qui transgresse leurs différences. Une photo annotée d'Egypte le montre bien (26 mars 1981, Denderah): autant l'opérateur entre dans le champ, mordant le cadre d'un bras et d'une jambe pour devenir un spectateur à la perspective décalée, autant le texte déborde l'image, la fait sortir de sa clôture pour relater la séquence de sa prise de vue ("J'ai attendu que tout le monde soit sorti et j’ai posé l'appareil par terre dans la poussière et la terre qui recouvre les dalles»), et indiquer les références stratifiées d'une démarche esthétique ("J'imite par ces 5 traits "l'Araignée mise au mur" de Francis Ponge, mais je pense aussi à Epstein et à Klee»).

L'écriture réalise donc un développement littéraire de l'image en rappelant les scansions temporelles de sa réalisation. Elle élabore aussi un récit défectif du sujet, qui se révèle toujours autre à lui-même dans l'aventure de son image - image préméditée, qu'il contemple désormais

Io. D. Roche, Photolalies, p. 5. 
extérieure à lui, dans sa différence. La photo-autobiographie poursuit par là une épiphanie intermédiale de soi, où le texte explore, dans le bord invisible et intime de la photo, le désir que le «moi» en a eu, le souvenir qu'il en a gardé, et la divergence que son regard mesure d'avec le scénario de la production. Entre cette projection du désir, cette césure de l'instantané, et cette méditation rétrospective, se construit en creux, dans les anachronismes de l'écriture et de l'image entrecroisées, l'ombre portée du sujet, sa part aveugle.

Si l'image constitue un embrayeur narratif, il s'agit toutefois davantage, chez Roche, d'un effet de récit que d'un véritable récit. La temporalité est certes dramatisée par une tension narrative dans les récits de prises de vue (entre imminence d'une préfiguration, crête de l'instant photographique et rêverie mémorative). Mais au-delà de la mise en récit de l'image, c'est la potentialité narrative dont l'image est porteuse que raconte vraiment l'écrivain-photographe, en abyme de la représentation. Son travail ne relate pas grand-chose du «moi»; il exhibe les actes manqués de l'opérateur où le rapport au réel se trouble. Denis Roche répugne d'ailleurs explicitement à narrer sa vie ou à publier des photos de famille, et nuance le terme "autobiographique" pour caractériser sa démarche ${ }^{11}$. En revanche, il amène le dispositif à se raconter lui-même. Le temps représenté dans ce double mode d'expression se montre informé par l'image: dans les brefs aphorismes qui s'étoilent autour des photos, c'est l'artificialité du temps narratif qui s'expose, son irréalité foncière, la fiction de son parcours en tous sens et de son étirement infini. Au vrai, la recherche rochienne sur l'imminence et le retard, sur la coupe du cadrage, sur la répétition rythmique d'une même séquence, sur l'inscription de l'opérateur dans le champ, relève bien plus de la poésie que du récit. Elle a beaucoup à voir avec le précédent travail de Denis Roche sur les bords du vers, sur la forme iconique du poème, sur le rythme discursif et les investissements imaginaires du sujet. Bien qu'il ait rompu spectaculairement avec la poésie décrétée «inadmissible» dans les années 1970, Roche reste lyrique dans sa mise en évidence des scansions qui préludent à l'image, comme dans les rimes temporelles de ses séries photographiques. Il reste métaphorique dans sa figuration dramatisée de la prise de vue comme épreuve de la mort. Métareprésentative comme sa poésie était métapoétique, sa photographie s'est récemment pourvue de

II. D. Roche, "Qu'est-ce qu'une photographie de famille?», p. 34. 
l'étiquette "interminable", où se prolonge l'écho sur "l'inadmissible" des débats d'avant-garde ${ }^{12}$. Sa photo-aubiographie est assez peu autofictionnelle, sinon sur ce mode second d'un enchantement distancié, parce qu'elle ne raconte guère le «moi», et elle n'est certainement pas romanesque - même si certaines planches-contacts sont assorties d'extraits de Louve basse, l'unique «roman» de l'écrivain. Mais est-ce un roman?

\section{La photobiographie de Gilles Mora: l'engouffrement narratif de la photographie}

La "photobiographie» que présente Gilles Mora deux ans plus tard (1983), infléchit nettement le nouveau genre vers le récit, non sans résonances avec l'autofiction, genre jumeau ${ }^{13}$. Dans son Manifeste photobiographique, Mora décrit en effet un mouvement collectif de photographes vers le support du livre, et vers l'épaisseur du texte, à rebours du «spectacle plat" de l'image tabulaire dans le photojournalisme ou dans le marché de l'art. L'intermédialité permet de renverser la fonction documentaire de la photo et de retourner la focale sur l'opérateur, pour le faire apparaître comme sujet. L'écriture œuvre l'invisible hors champ de la photo, dans une "exploration argentique du moi» ${ }^{14}$ :

Le texte a eu beau jeu, alors, de prendre sa revanche, par tous ses élargissements de fiction, d'imaginaires ou de fantasmes que paraissait désormais lui refuser l'image constat ${ }^{15}$.

Il ne s'agit pas seulement, pour le texte, de combler les absences de l'image en narrant l'expérience photographique, le «j'y étais» de l'opérateur. Il est aussi question de révéler la part subjective, intime, de toute photographie, comme "projection de notre espace privé sur le monde extérieur». De révéler la dimension autoportraitiste de toute prise de

I2. D. Roche, La photographie est interminable. Dans les premières pages, Roche expose les liens de son travail poétique sur les "antéfixes» et les débuts de sa photobiographie.

13. Je remonte aux origines communes de la photobiographie et de l'autofiction, autour de Roland Barthes par Roland Barthes, dans mon article, cité en bibliographie.

I4. G. Mora, «Photobiographies», p. 107.

I5. G. Mora, L'été dernier, p. 11 sq. 
vue: "Notre épiphanie sera autobiographique toujours» ${ }^{16}$, quelle que soit la scène représentée.

Lorsqu'il déplie la mémoire, le récit Deep South, qui accompagne et illustre le Manifeste, déploie l'image au-delà de sa visibilité, par le rappel de sensations nécessairement tues dans la photographie:

La seule sensation forte que me procurerait Little Rock, je n'allais même pas penser à la transformer en photographie. S'était immédiatement collée à moi, d'un coup, la chaleur humide de ce crépuscule rouge et l'odeur de vase du Golfe, remontant jusqu' ici ${ }^{17}$.

Ce que l'objectif ne peut saisir, ce qu'il a manqué dans la photo qui n'a pas été prise, le texte de Mora le restitue, avec une continuité narrative qui s'éloigne beaucoup du style saccadé de Denis Roche. L'écriture creuse l'image, dans un fantasme de pénétration qui double la surface photographique de toute une profondeur charnelle:

Le Deep South, lui, est un énorme vagin de noire dans lequel il faut s'enfoncer en se laissant faire. C'est une dépression visqueuse et chaude qui sauve le reste du pays de mort craquante par puritanisme et assèchement ${ }^{18}$.

Ces évocations métaphoriques restaurent la dimension existentielle des prises de vue, mais aussi la part d'aura qu'a perdue l'art photographique, au sens où Walter Benjamin définissait «l'aura» comme «l'apparition d'un lointain " ${ }^{19}$, ou comme "l'ensemble des images qui, surgies de la mémoire involontaire, tendent à se grouper autour [d'un objet] ${ }^{20}$. Les associations narratives du souvenir font apparaître la circonstance unique que l'image a refoulée pour advenir. Elles provoquent une épiphanie de ce qui, de la subjectivité, s'est évidé au cœur de la trace photographique, mais dont celle-ci conserve l'imperceptible halo révélé par l'écriture. En revanche, les espaces du texte et de l'image demeurent stables et distincts dans cet album photobiographique. Chez Denis Roche, l'opérateur devenu spectateur expose le texte aux questionnements de l'image,

\footnotetext{
I6. Ibid., p. 14.

17. G. Mora, «Deep South", in L'été dernier, p. 20.

I8. Ibid., p. 30.

19. W. Benjamin, Petite histoire de la photographie, p. 20.

20. W. Benjamin, «L'œuvre d'art à l'ère de sa reproductibilité technique», p. 178.
} 
se demande en quoi la photo le regarde, et transgresse les limites de chaque art par une dé-mesure réciproque de l'image et du langage. Chez Mora, le récit prend le pas sur l'hybridité du dispositif, et s'engouffre dans l'exploration d'un inconscient de la vision.

D'une "icône laconique» (Roche) à une «syntaxe iconique" (Mora), se constitue donc un genre qui s'élargit bientôt pour décrire et regrouper de nombreuses démarches contemporaines. En 1984, Mora dirige un collectif intitulé La Photobiographie dans les Cahiers de la photographie, qui fédère à cette enseigne des contributions de Claude Nori, Bernard Plossu, Raymond Depardon, Sophie Calle... Ce «tournant narratif» ${ }^{21}$ de la photographie se généralise dans l'édition, quelle que soit la qualité des diverses productions ${ }^{22}$, et diffuse dans les marges de l'autofiction. Tandis que la photobiographie se met ainsi en quête d'un texte fantôme susceptible de révéler dans l'image la subjectivité d'un regard et d'un moment vécu, l'autofiction, elle, se rapproche d'un imaginaire photographique et fonde souvent le récit de soi sur une «image fantôme» (Hervé Guibert), porteuse d'une inexprimable vérité personnelle, comme l'a bien montré Roger-Yves Roche dans Photofictions.

\section{Photoromans: Anne-Marie Garat}

C'est dans ce contexte de coalescence de la narration et de la photographie que se développe le travail littéraire et essayiste d'Anne-Marie Garat. Spécialiste des techniques de l'image, Anne-Marie Garat est publiée par Denis Roche éditeur dans la collection "Fiction \& Cie» (Seuil), pour deux de ses ouvrages (Photos de familles en 1994 et Dans la pente du toit en 1998). Elle commente aussi remarquablement la photolittérature rochienne ${ }^{23}$. Si cette proximité s'exprime au plan esthétique, c'est certainement dans la dimension légendaire accordée à la photographie.

2I. A ce propos, voir J. Baetens, "La lecture narrative de l'image photographique».

22. Dans un article intitulé "Pour en finir avec la photobiographie», Gilles Mora dénonce avec désenchantement les abus qu'a suscités, dans l'édition, une profusion d'ouvrages se réduisant à une "collection d'images» accompagnées d'un «maigre commentaire» autobiographique.

23. Anne-Marie Garat, "A Denis Roche». Voir aussi la participation conjointe d'Anne-Marie Garat et de Denis Roche au numéro spécial La Famille de la revue La Recherche photographique (1990). 


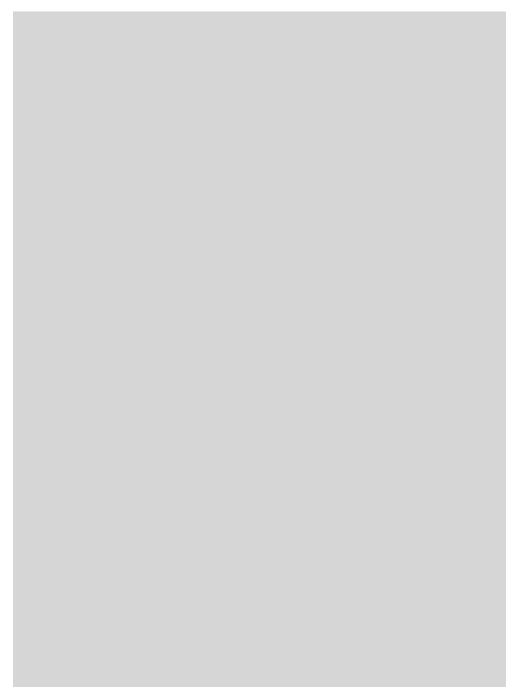

Fig. 3 - Anne-Marie Garat, collection particulière, Photos de familles. Un roman de l'album, Arles, Actes Sud, 2011.

$\mathrm{Au}$ "souvenir chimique» qui s'est déposé sur la pellicule, dans un instant unique, s'oppose chez Garat le souvenir narrativisé que chaque image suscite, pour son opérateur, son sujet ou son spectateur, et qui a toujours partie liée à l'imaginaire. Plus qu'elle ne fixe le temps, la photographie est elle-même emportée dans le flux temporel de ses manipulations techniques, de sa révélation progressive, de sa remémoration toujours reconstruite en fonction des enjeux du présent, et de ses multiples contemplations a posteriori ${ }^{24}$. Comme chez Roche, le «drame photographique» touche au temps et à la mort, et toute tentative d'"opérer sur le temps» par la photographie n'est que fiction. Mais pour Anne-Marie Garat, ce "drame " est matière à intrigue et sert de déclencheur à l'élaboration romanesque:

La photographie ménage des aires de liberté, des pauses et des silences, elle ne reproduit pas le temps réel, elle le transpose dans une durée autre, autonome, qui est celle de la création romanesque ${ }^{25}$.

Le dispositif rochien met en intrigue les antagonismes du temps et de l'espace dans l'acte photographique, mais il tend à interrompre la dérive du récit pour revenir à son hétérogénéité constitutive. Il montre comment l'opérateur entre dans la fiction, et comment il en sort lorsquapparaît la déhiscence entre successivité du vécu et spatialisation immédiate de l'image. La dissension des médias embraye et contrarie tout à la fois la narration, toujours autoréflexive. Chez Garat, la photobiographie devient l'instrument herméneutique d'une quête qui excède constamment son dispositif, dont les variantes imaginaires se développent en des univers

24. A ce propos, voir l'intéressant entretien de l'écrivaine avec Laurence Brogniez dans «Le cinéma dans l'œuvre d'Anne-Marie Garat».

25. A.-M. Garat, Photos de familles, p. 65. 
Fig. 4 - Anne-Marie Garat, collection particulière, Photos de familles, Paris, Seuil, 1994.

qui s'enchevêtrent à différents degrés de fiction. L'essai Photos de familles (1994) le montre bien. Cet ouvrage, qui se présente comme le livrejubilé de l'album photographique désormais centenaire, agence un discours d'histoire culturelle et sociale sur la photographie, des analyses d'images d'inconnus où se déploie la fiction autour des figures représentées, des récits et des documents autobiographiques, ainsi que des digressions autofictionnelles. Le glissement est parfois imperceptible, d'un commentaire sur des archives d'anonymes chinées aux puces, au récit de soi. Ainsi, une photo de famille est d'abord décrite comme un document social, dans toutes les incertitudes de son identification:

Voici des sœurs, campant un assemblage de six portraits en pied, adossées au mur de la ferme devant une porte close d'un gros crochet. Peut-être sur une route de montagne, des prés? ${ }^{26}$ 
Cette image, que rien ne différencie d'autres portraits de la culture populaire, se redéfinit, quelques pages plus loin, comme un document familial, au gré des dévoilements progressifs du récit:

Il n'est pas indifférent que je sache aussi que celle-ci s'exila en France, prit le voile et devint ensuite supérieure d'un couvent, à Lourdes. Que celle-ci épousa mon grand-père, rescapé de 14 , et qu'elle lui apprit à lire, à écrire ${ }^{27}$.

Mais ce n'est que Dans la pente du toit que cette histoire-là sera vraiment narrée, sur le mode autofictionnel.

En lieu et place d'images personnelles, le texte se détache parfois en blocs de petits caractères pour décrire une photo absente ("Je me souviens de l'instant où j'ai souri pour cette photographie" ${ }^{28}$ ) ou encore une photo qui n'a pas été prise ("Aucune photographie n’en rend compte» ${ }^{29}$ ), comme autant de médaillons de mots. Dans ces phénomènes de contamination entre les registres et les supports du récit, la photobiographie devient à la fois la biographie historique d'un art (« une histoire de gens ordinaires, de temps qui passe, de souvenir et d'oubli, de mort, d'amour " ${ }^{30}$ ); le récit du "roman social» à travers ses produits les plus intimes et les plus dévalués, les photos de famille dont aucun collectionneur ne veut; et l'histoire de soi. Une commune emprise de la fiction caractérise ces trois composantes du genre, autour de leurs manques respectifs. Quel vécu individuel fut celui de ces gens de peu, de la grande Histoire collective? Ces destinées obscures n'ont pas de mémoire, alors il faut les réinventer, et saisir dans la résistance à notre regard, dans la différence figée de ces images centenaires, le reflet des consciences privées qui ont subi les massacres de masse:

Alors inventons la légende. Ces yeux ont vu passer le siècle. Pas le nôtre, l'autre. Cette femme fut la contemporaine de tout le XIX'e siècle et de ses bouleversements. Imaginons qu'elle a pu voir, petite fille, défiler sous ses fenêtres les derniers soldats-enfants de la boucherie napoléonienne, les Marie-Louise partant au massacre. [...] Dans quelques mois, début août, elle entendra sonner le tocsin de la grande guerre, la «dernière».

\footnotetext{
27. Ibid., p. 124.

28. Ibid., p. 45.

29. Ibid., p. 154.

3o. Ibid., p. 7.
} 
1914. Mon grand-père a 25 ans. Mobilisé, il quitte ses vignes, il part. Militaire depuis trois ans, il fera quatre ans de plus. Il est illettré ou presque, il n'a pas vu beaucoup de pays, il va voyager. Il va perdre un oil, le pouce de sa main droite, c'est beaucoup pour un paysan ${ }^{31}$.

En fictionnalisant le point de vue du sujet photographié qui regarde l'opérateur, la photobiographe, comme si c'était l'Histoire qui venait la regarder, ranime la part aveugle des récits historiques, celle des tragédies individuelles, qu'elle relate désormais au présent aoristique. Elle revivifie leurs acteurs dont l'arrêt sur image se charge de tout un legs de noirceur, d'attente et de contamination mortifère ("elle a un œil fixe de poule, de belette, un œil carnassier, vitrifié et sauvage " ${ }^{32}$ ). Sa démarche s'apparente à une véritable enquête, lorsqu'elle s'arrête aux détails techniques de l'image, aux faiblesses de la mise au point ou de l'exposition, à tel flou, à tel noir, où se révèlent "ces choses qu'on ne voit pas dans le flux de la vie» ${ }^{33}$. C'est donc une pulsion scopique que met en œuvre la contemplation photobiographique, toujours prête, chez Garat, à se déployer en une intrigue romanesque, en une "soif de récits", pour imaginer les trajectoires singulières des personnages. Dans Chambre noire (1990), roman contemporain de Photos de familles, le personnage de Milena, photographe vouée, comme son prénom l'indique (puisque issu d'une racine grecque signifiant «noir»), au travail au noir de la mémoire des images, élucide ainsi, à partir d'un repérage technique très précis, le mystère d'une série de photographies presque identiques prises dans le domaine de sa belle-famille. Le relevé de différences minimes dans les images l'amène à reconstituer le point de vue de l'opérateur, jusqu'à retrouver le plan exact de la prise de vue, et creusée dans la terre, l'empreinte du trépied photographique. Là encore, ce sont les particularités du support qui révèlent un secret existentiel, et à travers lui, le secret d'une mort sans histoire, le drame d'un personnage tombé en 1914 sans vivre la guerre autrement que par émanation du mal:

3I. Ibid., p. 16 sq.

32. Ibid., p. 17.

33. A ce propos, voir l'article de M. Verthuy, qui fait le rapprochement avec le film Blow-Up. 
C'est une image plate, sans un reflet, un éclat. Un travail superbe des valeurs de gris, déclinées en nuances subtiles, mais mates, éteintes. [...] L'oil s'égare, enquête, se perd, rejoint un centre qui n'est pas central, ne rencontre que la répétition vaine du motif végétal, la ligne brisée, perdue, la courbe, escamotée, des amorces, des brisures fulgurantes, sans fin, sans raison. [...] Seule constante, la ponctuation de l'épine, dardée du noir profond, l'épine aiguë. Tout cela à une distance telle qu'on n'est plus au spectacle, mais prisonnier d'un plan si rapproché qu'il n'y a plus de fuite possible, nulle échappée. Monde clos, d'asphyxie mentale. Image monstrueuse de la mort. Le chaos. La guerre ${ }^{34}$.

Dans Nous nous connaissons déjà (2003), la narratrice sans nom est chargée d'identifier une scène de boucherie dont des photographies ont été retrouvées dans les archives d'un château de Gironde. Elle découvrira que les images documentent un viol au cœur des Pyrénées, dans une gare au décor fantastique, au cours des fuites de miliciens de la Seconde Guerre mondiale. L'horreur du sexe exhibé, la monstration clinique du cadavre torturé, qu'imposent les photographies amènent l'enquêtrice à épouser la perspective voyeuse de l'opérateur. Ce qui se donne à voir alors, dans les fictions de l'œil, c'est l'innommable de l'Histoire qui déchire le regard:

[...] il m'apparaissait que l'ordonnateur, le scénographe et le dramaturge inspiré de cette boucherie était le photographe lui-même, le grand absent de toutes ces photos, dont nulle ombre portée, nulle trace de lui n'apparaissait, mais vers qui convergeaient les regards, pour qui posaient avec docilité, servilité, les acteurs sous contrôle ${ }^{35}$.

De l'invisible dans l'image aux non-dits de l'Histoire, et au mal immémorable qui traumatise des particuliers sans histoire, le défaut des récits collectifs, les secrets de famille et les infirmités personnelles se répondent, qui ne peuvent s'élaborer que par l'imaginaire:

[...] entre ses images, il y a le non-dit sauvage de l'existence, il y a des histoires, innombrables. Et dans l'image elle-même, souvent, dans le

34. A.-M. Garat, Chambre noire, p. 102 sq.

35. A.-M. Garat, Nous nous connaissons déjà, p. 118 sq. Pour une belle lecture de ce roman, voir C. Rodgers, "Quête féminine dans Nous nous connaissons déjà d'AnneMarie Garat». 
secret des visages et des lieux, les destins, la matière noire du roman. Par accident, pour peu qu'on sache regarder, qu'on apprenne à regarder. Histoire des êtres, histoire de la photographie. Dans les photographies et entre elles, dans leur silence, s'engouffre la fiction $[\ldots]^{36}$.

Par là, ce sont deux fictions fondatrices qui se narrent entre les lignes des intrigues garatiennes. D'une part, le fantasme d'une fusion des êtres par le biais de l'image, à rebours d'une fusion des corps toujours problématique dans cette œuvre. Ainsi Milena, choquée par le secret de sa naissance, qu'elle ressasse dans la "chambre noire» de son esprit comme issue d'un viol, ne communique-t-elle avec les autres que par l'entremise de son objectif photographique, porté à même le ventre: "elle photographie avec sa peau, pas avec son oil »37. Mais son ventre de femme demeure stérile et frigide, coupé d'un véritable contact des chairs: «un ventre noir, sans enfants ». En rêvant cette interpénétration des consciences à partir des positions et des circonstances de la prise de vue, Anne-Marie Garat met toujours en évidence la fragilité de l'identité personnelle, la difficile singularisation de l'individu au sein d'une lignée, et d'une histoire collective. Entre les meurtres de masse, le roman familial transpersonnel et l'état de conscience privé, c'est la gémellité des destins et la dissolution tragique de la personne qui s'éprouvent ${ }^{38}$. La photographie revêt alors une fonction compensatrice: en narrant la perspective des acteurs de l'image, en leur prêtant voix dans des monologues au discours indirect libre, le récit garatien postule que voir comme l'autre permettrait d'être comme l'autre, de nier l'écart existentiel qui sépare les individus. L'image devient la métaphore visuelle de cet abîme qu'est l'autre; et de ce vertige identitaire, la narration procède à une démarche réparatrice où l'histoire d'un regard se fait l'histoire d'une reconnaissance de soi. "Je veux atteindre cet endroit le plus noir, je veux mettre la lumière à mal, lui faire mal», dit Milena, qui finit pourtant, en orchestrant la photo de famille conclusive de son parcours romanesque, par assigner à chacun sa place, à chaque individu sa différence:

36. A.-M. Garat, Photos de familles, p. 36.

37. A.-M. Garat, Chambre noire, p. 141.

38. Sur la thématique du double chez Garat, voir les deux articles de C. Rodgers cités dans la bibliographie. 
Aujourd'hui, dit Milena, je vais prendre une photographie de la famille. Sur la terrasse, après le déjeuner, je les installerai tous comme on le faisait autrefois. [...] Mais je veux aussi les fissures, les failles, un rang inachevé, le nombre impair ${ }^{39}$.

Si elle fonde une poétique du récit ${ }^{40}$, la séquence d'une reconnaissance suppose aussi, chez Garat, une fiction plus intime encore, qui tient à une conception commune de l'image et de la sensibilité. Les âmes peuvent s'épouser, se confondre et se réidentifier à travers la contemplation photographique, parce que l'événement s'impressionne de manière similaire, sur la pellicule et dans la perception subjective. La métaphore du nuage, récurrente chez Garat, désigne cet essaim de particules lumineuses qui irradient la gélatine du film photographique; mais encore cet "air de famille» impalpable, issu d'une séquence génétique, qui s'imprègne d'une génération à l'autre, par-delà les personnalités, et qui dessine sur les visages d'une lignée le «patron» de drames ancestraux et refoulés; enfin, cette nuée des faits qui arrivent et qui capturent les individus dans la nasse de l'Histoire, à l'instar du nuage atomique libéré par la catastrophe de Tchernobyl, qui constitue la trame de la photo de famille à la fin de Chambre noire. Toutes ces contaminations indiscernables, qui minent l'identité personnelle, trouvent leur répondant dans le pouvoir quasi magique de communication auratique qu'attribue la romancière à la photographie. L'une de ses héroïnes, Laura (ou l'aura, dans Nous nous connaissons déjà) reconstitue les bribes d'un récit de meurtre qui a fait basculer son enfance. Sa rencontre avec la narratrice spécialiste de photographie révèle le souvenir fulgurant d'une communion des âmes dans l'imminence de la mort: "Voilà mon innocence et la sienne, ou notre crime ensemble, d'avoir conçu sur la plage de notre enfance ce lien indéfectible " ${ }^{41}$.

Du nuage du multiple à l'impression unique dans la conscience personnelle, du divers et de l'hétérogène au tressage d'une histoire de soi, la métaphore du nuage condense les antagonismes de la dramatisation garatienne. Elle fait de l'image, ce nuage de points lumineux devenu

39. A.-M. Garat, Chambre noire, p. 263 sq.

40. Dans le riche entretien de Danièle Méaux avec Anne-Marie Garat (in Photographie et romanesque), les techniques romanesques qui rencontrent la dramatisation photographique sont abordées du point de vue de la critique et de l'écrivaine.

4I. A.-M. Garat, Nous nous connaissons déjà, p. 327. 
forme, le support où les oppositions structurales de la mort et de la vie, du moi et des autres, du présent et de l'Histoire, s'enchevêtrent en un réseau narratif:

Il lui faut aller voir derrière, à l'envers symétrique, que la nuit soit le jour, que la neige soit noire. Marcher dans la nuit blanche de l'enfance où les flocons noirs rayent la pellicule de l'air ${ }^{42}$.

De cet air qui s'impressionne sur le négatif photographique, à l'air de famille qui s'en dégage en des formes reconnaissables, c'est, au plus profond, les abîmes de soi que cherche à voir la photobiographe. Organisé thématiquement comme un traité typologique (albums, portraits, etc.), Photos de familles consacre en effet l'un de ses chapitres à un récit singulier, parce qu'entièrement autobiographique, comme l'indique l'article défini de son intitulé: "Le Voitgländer». L'absolu individuel voisine donc avec les dizaines de "vies minuscules" que regroupe l'essai. Autour de la description du vieil appareil photographique de sa famille, que seul le père avait le droit de manipuler, vu le "caprice extrême du déclencheur et la complexion réputée fragile du système de réglage», se narre toute une scène primitive dans un fantasme fétichiste. S’emparer du Voitgländer, pour la narratrice, c'est s'approprier quelque chose du désir du père, qui lui reste interdit: «Je pressentais bien que sa jouissance conférait une trop grande autorité à mes parents pour qu'ils en partagent la magie " ${ }^{43}$. La photographie qui n'aura pas été prise d'une pêche miraculeuse de l'enfance, l'image désirée d'une prise extraordinaire d'anguilles noires et velues se contorsionnant dans leur eau visqueuse, expose alors le récit à la représentation métaphorique d'une "Méduse». Y affleure la vision aveuglante de la mère phallique. Cette rêverie œedipienne aboutit toutefois à un dépassement du traumatisme enfantin: ici encore, le texte se substitue à une photographie, pour se détacher en une vignette de mots. Dans cet aparté, se dit le désir de voir, en deçà de soi, sa propre origine, et la possibilité de réoriginer par le biais du langage, à partir de la photographie interdite:

J'assiste au spectacle qui condense cet après-midi d'été en un cadre parfait, un instant de pure ressemblance. Au centre de la photographie interdite ${ }^{44}$.

42. A.-M. Garat, Chambre noire, p. 245.

43. A.-M. Garat, Photos de familles, p. 49.

44. Ibid., p. 52. 
La relève de l'imaginaire par le symbolique est aussi une relève de l'image manquante par le récit littéraire, qui redonne naissance au «moi» des mots. C'est par l'élaboration narrative des images défectives, dans le simulacre de la représentation, que peut se définir le sujet.

De Denis Roche à Anne-Marie Garat, de la photo-aubiographie au photoroman, ce sont donc des renversements de postures qui s'imposent. Alors que Roche fait toujours intrusion dans l'image, qu'il pénètre dans le champ comme pour étreindre l'instant d'une vie, et posséder l'autre désiré dans un rituel de «balisage amoureux», Garat, elle, explore un imaginaire matriciel de la délivrance d'une histoire latente, à travers les lambeaux d'un récit décousu, social, familial ou personnel. Roche s'affronte à l'immédiateté de la prise de vue, et ne s'intéresse guère au tirage qu'il ne pratique d'ailleurs pas du tout. Garat évalue la durée d'une révélation progressive de l'image, qu'elle transpose en un processus de dévoilement romanesque: une maïeutique de soi. Chez l'un et l'autre, toutefois, c'est le cadrage de la représentation qui toujours jugule l'engouffrement narratif. Roche désigne par le texte les limites d'une "chambre blanche" photographique que vient circonscrire l'image. A l'inverse, Garat charge le texte de cadrer la mémoire subjective en lieu et place de l'image, de découper dans son flou, et d'arrêter les dérives de l'affabulation mémorative autour des émanations auratiques de la photographie, par une suture de mots. Ainsi, Chambre noire se structure d'abord et surtout comme un montage verbal, formulaire: de l'en-tête du premier chapitre aux ultima verba du roman, c'est la même clausule qui revient, et qui clôt le texte comme on cadre une image. Chaque chapitre se construit sur la reprise des derniers mots du chapitre précédent, pour aboutir à un tout fermé. La littérature se fait par là l'épreuve vivante de la photographie, qui ranime et situe ses enjeux affectifs pour le sujet contemplatif. C'est aussi ce qui met à distance les vertiges du regard, donne contour aux nuances instables de la vie, impose une forme pensable aux mouvements fluctuants des nuages du réel.

Dominique Kunz Westerhoff Université de Lausanne 


\section{BIBLIOGRAPHIE}

\section{Textes}

Benjamin, Walter, "L'œuvre d'art à l'ère de sa reproductibilité technique» (1936), in Euvres II, tr. Maurice de Gandillac, Paris, Denoël, 1971, p. 171-210.

—, «Petite histoire de la photographie» (1931), tr. André Gunthert, Etudes photographiques, 1 (novembre 1996), en ligne http://etudesphotographiques.revues.org/99.

Garat, Anne-Marie, "Images en famille», La famille. La recherche photographique, 8 (février 1990), p. 29-31.

—, Chambre noire, Arles, Actes Sud, coll. Babel, 2008 [1990].

—, Photos de familles, Paris, Seuil, 1994 (nouvelle édition augmentée et réactualisée sous le titre Photos de familles. Un roman de l'album, Arles, Actes Sud, 2011).

—, Dans la pente du toit, Paris, Seuil, 1998.

—, "A Denis Roche», in Denis Roche. Pour voir les photographies, 11 (2001), p. 45.

—, Nous nous connaissons déjà, Arles, Actes Sud, 2003.

Mora, Gilles, La Photobiographie. Les Cahiers de la photographie, 13 (1984).

—, «Photobiographies» (1999), in Traces photographiques, traces autobiographiques, éd. par Danièle Méaux, Jean-Bernard Vray, SaintEtienne, Publications de l'Université de Saint-Etienne, 2004, p. 107-113.

—, "Pour en finir avec la photobiographie», in Traces photographiques, traces autobiographiques, éd. par Danièle Méaux, Jean-Bernard Vray, Saint-Etienne, Publications de l'Université de Saint-Etienne, 2004, p. 115-117.

Mora, Gilles, Nori, Claude, L'été dernier. Manifeste photobiographique, Paris, Editions de l'Etoile, 1983. 
Roche, Denis, Légendes de Denis Roche. Essai de photo-autobiographie, Montpellier, Gris Banal Editeur, 1981.

—, La disparition des lucioles (réflexions sur l'acte photographique), Paris, Editions de l'Etoile, 1982.

—, Ecrits momentanés. Chroniques photo du magazine City (1984-1987), Paris, Audiovisuel, 1988.

-, Photolalies. Doubles, doublets et redoublés, Paris, Editions Argraphie, 1988.

—, "Qu'est-ce qu'une photographie de famille?», La famille. La recherche photographique 8 (février 1990), p. 34-35.

—, Le Boîtier de mélancolie. La photographie en 100 photographies, Paris, Hazan, 1999.

—, La photographie est interminable. Entretien avec Gilles Mora, Paris, Seuil, 2007.

\section{Etudes}

Baetens, Jan, "Légendes de Denis Roche: un livre "incompatible"?», in Denis Roche: l'un écrit, l'autre photographie, éd. par Luigi Magno Lyon, ENS Editions, 2007, p. 69-83.

—, "La lecture narrative de l'image photographique», in Jean-Pierre Montier, Liliane Louvel, Danièle Méaux et Philippe Ortel, Littérature et Photographie, Rennes, Presses Universitaires de Rennes, 2008, p. 339-348.

Brogniez, Laurence, "Le cinéma dans l'œuvre d'Anne-Marie Garat", Contemporary French and francophone Studies, 9/3 (2005), p. 227-235.

Buisine, Alain, "Tel Orphée...", Photolittérature. Revue des sciences humaines, 210 (1988), p. 124-149.

Calle-Gruber, Mireille, «Ecrit avec l'image. Sur un essai photo-autobiographique: Légendes de Denis Roche", Les Cahiers de la photographie. Denis Roche, 23 (1984), p. 29-42.

Kunz Westerhoff, Dominique, «Photobiographie, autofiction, photofiction: Les Années d'Annie Ernaux", in L'Autofiction: variations génériques et discursives, Bruxelles, Academia/L'Harmattan, 2012, p. $147-170$. 
MÉAux, Danièle, «La photographie à l'épreuve du roman. Entretien avec Anne-Marie Garat", in Photographie et romanesque, éd. par Danièle Méaux, Etudes romanesques, 10 (2006), p. 333-344.

MÉaux, Danièle, VRay, Jean-Bernard (éds), Traces photographiques, traces autobiographiques, Saint-Etienne, Publications de l'Université de Saint-Etienne, 2004.

Roche, Roger-Yves, Photofictions. Perec, Modiano, Duras, Goldschmidt, Barthes, Villeneuve d'Asq, Presses universitaires du Septentrion, 2009.

Rodgers, Catherine, "Quête féminine dans Nous nous connaissons déjà d'Anne-Marie Garat», Dalhousie French Studies, 73 (Winter 2005), p. 139-150.

—, "Dans la Pente du toit d'Anne-Marie Garat: élaboration, déconstruction du conte familial", in Affaires de famille. The Family in Contemporary French Culture and Theory, ed. by Marie-Claire Barnet, Edward Welch, Amsterdam/New York, Rodopi, 2007, p. 83-103.

Verthuy, Maïr, "Anne-Marie Garat et la photographie, ou Blow-Up revisité", Art and Contemporary Prose, ed. by Michaël Bishop, Dalhousie French Studies, 31 (1995), p. 97-108.

\section{Crédits iconographiques}

Fig. 1-2:

Tous droits réservés.

Fig. 3-4:

Avec l'aimable autorisation de l'auteur. 